\title{
Solar origin of in-situ near-relativistic electron spikes observed with SEPT/STEREO
}

\author{
A. Klassen ${ }^{1}$, R. Gómez-Herrero ${ }^{1,2}$, B. Heber ${ }^{1}$, Y. Kartavykh ${ }^{3,4}$, W. Dröge ${ }^{3}$, and K.-L. Klein ${ }^{5}$ \\ 1 Institut für Experimentelle und Angewandte Physik, Universität Kiel, Germany \\ e-mail: klassen@physik.uni-kiel.de \\ 2 Space Research Group, University of Alcalá, Spain \\ 3 Institut für Theoretische Physik und Astrophysik, Universität Würzburg, Germany \\ 4 Ioffe Physical-Technical Institute, St. Petersburg 194021, Russia \\ 5 Observatoire de Paris, LESIA, CNRS-UMR 8109, 92195 Meudon, France
}

Received 12 December 2011 / Accepted 12 April 2012

\section{ABSTRACT}

\begin{abstract}
During 2010-2011 the Solar Electron Proton Telescope (SEPT) onboard the twin STEREO spacecraft detected a number of typical impulsive electron events showing a prompt intensity onset followed by a long decay, as well as several near-relativistic so-called electron spike events. These spikes are characterized by a very short duration of below 10-20 min at FWHM, almost symmetric time profiles, velocity dispersion and strong anisotropy, revealing a very weak scattering during particle propagation from the Sun to STEREO. Spikes are detected at energies below $300 \mathrm{keV}$ and appear simulateneously with type III radio bursts detected by SWAVES/STEREO and narrow EUV jets in active regions. Using particle, EUV and radio imaging observations we found that nearrelativistic electrons were accelerated simultaneously and at the same location as the electrons emitting the accompanying type III radio bursts and together with coronal EUV jets. Furthermore, the sources of type III radio bursts match very well the locations and the trajectories of the associated EUV jet. Applying a particle propagation model we demonstrate that the spike characteristics reflect both, properties of the accelerator and effects of interplanetary propagation.
\end{abstract}

Key words. Sun: particle emission - Sun: flares - Sun: radio radiation - Sun: UV radiation - interplanetary medium acceleration of particles

\section{Introduction}

Impulsive near-relativistic electron events observed in interplanetary space are commonly associated with solar flares and they are very often accompanied by type III radio bursts (Lin 1985; Kahler et al. 2007). Type III bursts are radio tracers of electron beams propagating from the flare site through the corona and the interplanetary (IP) medium (e.g. Suzuki \& Dulk 1985).

Electron spike events and typical impulsive electron events have not only some common characteristics such as velocity dispersion, a prompt intensity increase and strong anisotropy during the onset time. They show also clear differences: the spikes are characterized by a very short duration of less than 10-20 min at full width at half maximum (FWHM), almost symmetric time profiles, and strong anisotropy during the whole event, revealing nearly "scatter-free" particle propagation from the Sun to the spacecraft (Lin 1974; Haggerty \& Roelof 2009; Klassen et al. 2011).

A close temporal and spatial coincidence between type III bursts and coronal jets in the EUV and X-ray ranges was already pointed out by Aurass et al. (1994), Raulin et al. (1996), and Innes et al. (2011). It was suggested that particle acceleration and jet formation occur at reconnection sites along the current sheets located between an emerging flux and preexisting open field (Shibata et al. 1992; Shimojo et al. 1996; Krucker et al. 2011).

Klassen et al. (2011) found that spikes show a very close temporal correlation with EUV jets and type III bursts and suggested that associated near-relativistic interplanetary (IP) electrons are accelerated at the same time and at the same place as coronal EUV jets and electrons emitting the accompanying type III bursts. Close temporal associations of typical impulsive electron events and especially ${ }^{3} \mathrm{He}$-rich particle events with jets (X-ray, or EUV) also had been discussed in the last decade e.g. by Wang et al. (2006) and Nitta et al. (2006).

In this paper, we extend the results of Klassen et al. (2011), and present new observations which show that spikes, decimetric-metric type III radio bursts and coronal jets are not only temporally correlated, but also located at the same place in the solar corona. The latter suggests a common source of spike electrons, coronal EUV jets and electrons emitting the accompanying type III bursts. Furthermore, applying the particle propagation model we show that the spike characteristics reflect both, properties of the accelerator and effects of interplanetary propagation.

\section{Instruments and data selection}

For our study we have selected a sequence of electron spikes on 26 February 2011 and an isolated spike on 19 March 2011 which were detected by the Solar Electron and Proton Telescope (SEPT) oboard the twin STEREO spacecraft. The event on 19 March 2011 was chosen due to the availability of Nançay Radioheliograph (NRH) (Kerdraon \& Delouis 1997) observations, allowing the sources of the accompanying type III radio bursts to be localized.

SEPT consists of two dual double-ended magnet/foil particle telescopes which measure electrons in the nominal energy 
range from 30 to $400 \mathrm{keV}$ and ions from 60 to $7000 \mathrm{keV}$ with an energy resolution of $\simeq 10 \%$ below $1200 \mathrm{keV}$ (Müller-Mellin et al. 2008). Both STEREO are non-spinning spacecraft, and the pitch-angle distributions of the detected particles on each s/c are provided by four separate telescopes: two of them are directed in ecliptic plane along the nominal Parker field both towards and away from the Sun (i.e. $45^{\circ}$ and $225^{\circ}$ west of the spacecraft Sun line, respectively), and the other two are directed towards the north and south perpendicular to the ecliptic plane. The electron sensor is covered by a thin foil which stops protons with energies $\leq 400 \mathrm{keV}$ but does not change substantially the electron spectrum. The magnet sweeps away electrons but lets ions pass. The geometry factor for each electron and proton telescope is $0.13 \mathrm{~cm}^{2}$ sr and $0.17 \mathrm{~cm}^{2} \mathrm{sr}$, respectively. The time resolution is $1 \mathrm{~min}$ and the field of view (FOV) is $52^{\circ}$.

For our analysis we used PLASTIC solar wind data (Galvin et al. 2008), SWAVES radio emission spectra (Bougeret et al. 2008), EUV images from SECCHI-EUVI (Howard et al. 2008), and MAG data (Acuña et al. 2008) from instruments onboard STEREO-A and EUV images from AIA onboard the Solar Dynamic Obsevatory (SDO) (Lemen et al. 2011).

\section{Observations}

\subsection{Electron spikes, type III radio bursts and jets: the 26 February 2011 events}

Figure 1 presents an example of a series of eight in-situ electron spikes observed by SEPT/STEREO-A on 26 February 2011 during a period of $16 \mathrm{~h}$. At this time the s/c was at a distance of $0.96 \mathrm{AU}$ from the Sun and $87^{\circ}$ ahead of the Earth on its orbit. All spikes were detected at energies below $300 \mathrm{keV}$, showing velocity dispersion and durations at FWHM shorter than 12 min. During the whole day the IP conditions were quiet with slow solar wind speed $V_{\mathrm{sw}} \simeq 300 \mathrm{~km} \mathrm{~s}^{-1}$, low density $\leq 2 \mathrm{~cm}^{-3}$, and low proton temperature between $1 \times 10^{4}$ and $1 \times 10^{5} \mathrm{~K}$.

The top panel shows the SWAVES dynamic radio spectrum in the frequency range $16-0.003 \mathrm{MHz}$. The electron spike time profiles in the energy range $55-65 \mathrm{keV}$ measured with telescopes looking towards the Sun (SEPT_Sun, blue line) and in the antiSun directions (SEPT_AntiSun, black line) are presented in the second panel. The bottom panel shows the spike dynamic energy spectrum in the energy range $35-500 \mathrm{keV}$. Thereby the time profiles of measured in-situ electrons and the energy spectrum (middle and bottom panels) were shifted by 13 min to take into account the difference between the propagation time of electrons along the nominal IP magnetic field lines and type III radio emission.

Apparently the sequence of all eight spikes streaming outwards from the Sun was associated with a series of bright type III bursts generated by beams of low energy electrons emanating from the corona during the same time interval. The type III bursts drift from high frequencies to the local plasma frequency around $0.02 \mathrm{MHz}$. The series of sharp-bursty radio emission increases at the frequencies between $0.01-0.03 \mathrm{MHz}$ at the low boundary of type III bursts numbers 5, 6, and 8 could be interpreted as Langmuir waves locally generated by the arrival of $2-12 \mathrm{keV}$ electron beams responsible for type III bursts (e.g. Ergun et al. 1998).

The vertical dashed lines (Fig. 1, middle panel) indicate the onset time of type III bursts and show a delay of 3-15 min between the type III radio burst and the spike onset. The last spike (number 8) which will be discussed later in Sect. 3.3 has a delay of $10 \mathrm{~min}$. Different reasons for such delays were discussed in a

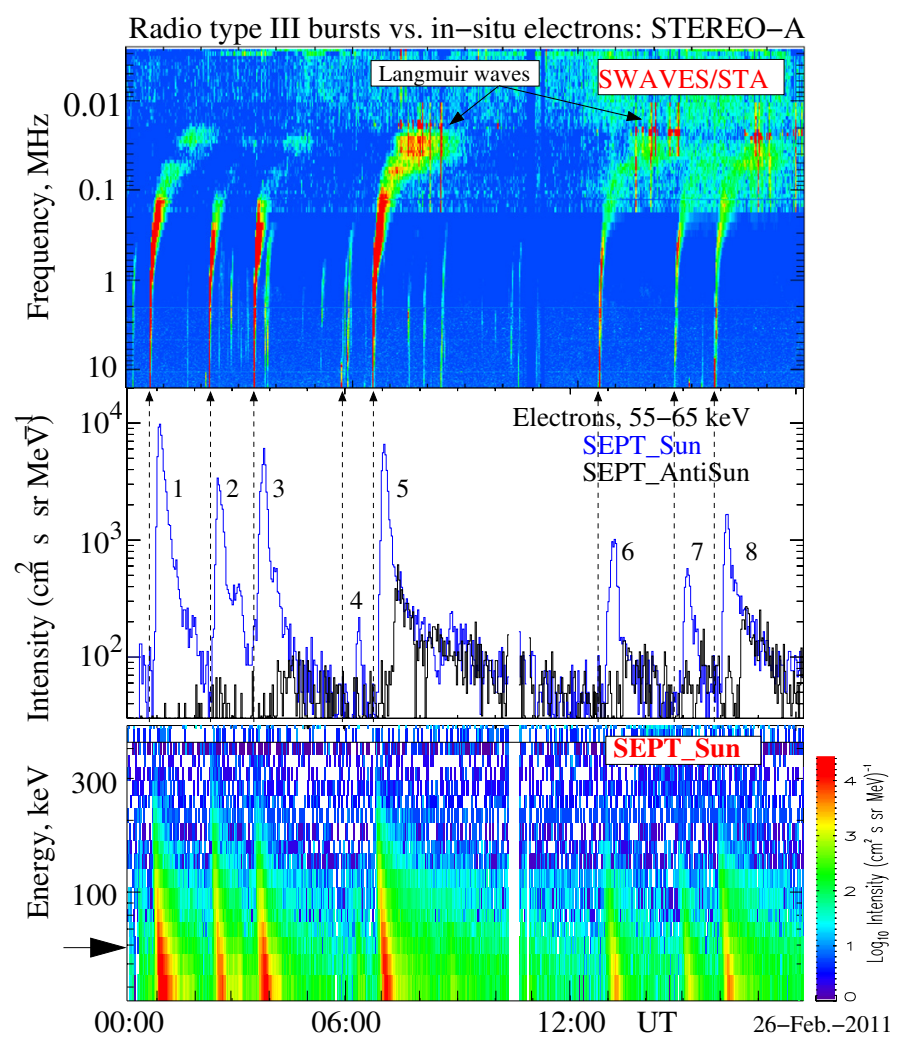

Fig. 1. Events on 26 February 2011: a sequence of type III bursts and electron spike events. Top: dynamic radio spectrum of type III bursts (SWAVES/STA). Middle: time profiles of electron spikes intensities in the energy range 55-65 keV measured with telescopes looking towards the Sun (SEPT_Sun, blue line) and in the anti-Sun directions (SEPT_AntiSun). The vertical lines indicate the onset of type IIIs relative to in-situ electron spikes. The numbers indicate the consecutive spikes. Bottom: dynamic energy spectrum of electrons in the energy range $35-500 \mathrm{keV}$. The time profiles of electrons and the energy spectrum is shifted by 13 min (middle and bottom panels) to take into account the the difference between the propagation time of electrons and type III radio emission. The arrow on the left indicates the $55-65 \mathrm{keV}$ energy channel.

number of papers (e.g. Krucker et al. 1999; Klassen et al. 2002; Kahler et al. 2007). In our case the time delays can be explained rather by a considerable (factor of 1.3-1.6) deviation of the magnetic field line length from its nominal value due to large scale turbulence in the solar wind (Ragot 2006; Kahler et al. 2011). There is no evidence for injections, apart from type III bursts, like shock acceleration (type II radio bursts, Haggerty \& Roelof 2002) or new radio sources in the low corona (Klein et al. 2005) which could be responsible for later electron injection, observed.

Sometimes spikes have double peaks (e.g. spikes numbers 2 and 3). In this case they are correlated with type III radio bursts, which also show a double structure - a bright type III radio burst followed by a weak type III burst. This means that an additional injection of near-relativistic electrons in interplanetary space has occurred.

Usually in spikes the main particle flux is detected by the telescope looking towards the Sun along the nominal magnetic field line (SEPT_Sun, blue line in Fig. 1), while the telescope looking in the opposite direction shows no intensity increase. That implies a strongly anisotropic and nearly scatter-free electron propagation from the Sun to the spacecraft.

Sometimes electrons streaming towards the Sun are also detected as spikes. Figure 1 (middle panel) shows peaks detected 
by the anti-Sun facing telescope in spikes No. 3, 5, 6, 7, 8 (black line). They are separated from the main peak registered by the Sun-looking telescope (blue line) by $\sim 20-30 \mathrm{~min}$, consistent with a reflecting boundary at approximately $0.5-0.7 \mathrm{AU}$ beyond the spacecraft along the magnetic field line, or $\sim 0.3 \mathrm{AU}$ in radial direction. This reflecting boundary must be very efficient, because of a relatively high (0.10-0.17) ratio between maximal intensities of outward and inward streaming electrons. Consequently, in spike No. 5 a velocity dispersion was observed also for the inward-streaming electrons. In total, the duration of this peak at FWHM was 13 min, which is longer than the duration of the main peak ( 9 min). Similar impulsive events exhibiting a separate peak of inward streaming electrons were discussed in papers by Agueda et al. (2010) and Wang et al. (2011). They have concluded that a reflecting boundary was located at a distance of $\geq 0.2-1.7 \mathrm{AU}$ along the magnetic field line beyond the Earth.

Klassen et al. (2011) found that type III radio bursts, shortlived ( $<5 \mathrm{~min}$ ) EUV jets and electron spikes show a very close one-to-one correlation with each other in time. This conclusion was achieved due to relatively high EUVI/STEREO image cadence of $\leq 2-5 \mathrm{~min}$. Unfortunately, for some of the presented spikes on 26 February a similar investigation was not possible, because EUVI images had a lower time resolution of 5-10 min, and some of type III bursts/spikes coincided with jets observed simultaneously in two different active regions close to the west limb. Moreover the jets and the corresponding type III radio burst occurred far behind the solar west limb (from the Earth's point of view) and were totally occulted by the solar disk which made it impossible to use the Nançay Radioheliograph (NRH) data to identify their spatial location.

\subsection{Spatial correlation of the spike acceleration source, jet and type III radio burst: event on 19 March 2011}

In contrast to the spikes discussed above, the event on 19 March 2011 was also observed by NRH that allowed us to localize the coronal metric radio sources of accompanying type III radio bursts and consequently the location of type III emitting electrons. Assuming that the type III radio bursts emitting low energy electrons and the spike's near-relativistic electrons belong to the same population we can conclude that the spike source is the same as the source of type III radio bursts.

This anisotropic beam-like electron spike was associated with a double type III burst (Fig. 2) and a coronal jet as observed first with EUVI/STA between 09:36 and 09:43 UT (Fig. 3) and then a few minutes later with AIA/SDO at 09:43-10:30 UT (Fig. 4). The EUV jet(s) (Carrington longitude $=65^{\circ}$ ) occurred in AR 11169 crossing the west limb from the Earth's point of view on 17 March. On 19 March this region was already 25 degrees behind the limb and at W20N22 as seen from STA. Due to this occultation SDO was able to observe the jet not from the beginning on but a few minutes later after the real jet onset. Similar as for the events on 26 February, the IP conditions were relatively quiet: $V_{\mathrm{sw}}=405 \mathrm{~km} \mathrm{~s}^{-1}$, a density of $1.5 \mathrm{~cm}^{-3}$, and a proton temperature $\leq 1.5 \times 10^{5} \mathrm{~K}$.

The top panel of Fig. 2 shows the SWAVES dynamic radio spectrum in the frequency range $16-0.003 \mathrm{MHz}$ with a doubled type III burst implying two successive injections of electrons into the IP medium. The middle panel shows the electron spike time profiles in the energy range 55-65 keV measured with telescopes looking towards the Sun (SEPT_Sun, blue line) and in the anti-Sun directions (SEPT_AntiSun, black line). The bottom panel shows the spike dynamic energy spectrum in the

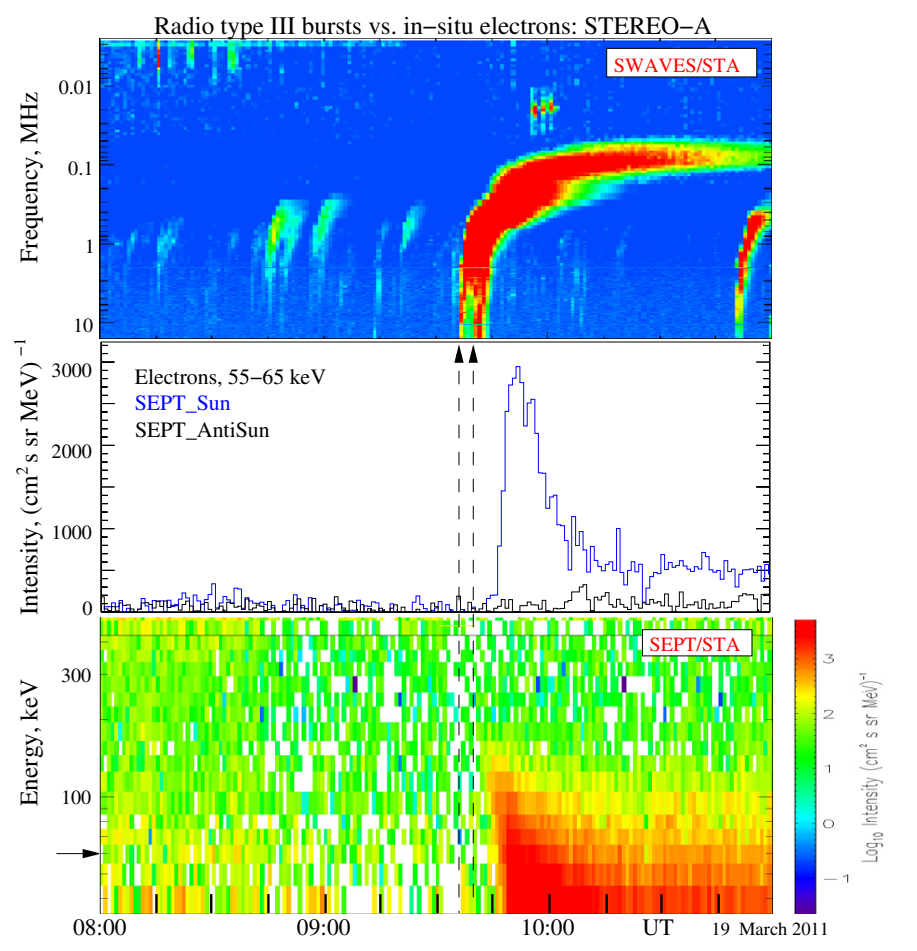

Fig. 2. Event on 19 March 2011. The same format as in Fig. 1. The time profiles and the energy spectrum is shifted by $12.5 \mathrm{~min}$ (middle and bottom panels) to take into account the the difference between the propagation time of electrons and type III radio emission. The arrow on the left indicates the 55-65 keV channel.

range 35-500 keV. Again the time profiles and the energy spectrum (bottom panel) were shifted so that it gives the arrival time in UT minus $12.5 \mathrm{~min}$. A value of $12.5 \mathrm{~min}$ is the difference between the propagation time of electrons along the nominal Parker field line $(20.5 \mathrm{~min})$ and the propagation time for the type III radio emission ( $8 \mathrm{~min}$ ). Making use of the solar wind speed of $405 \mathrm{~km} \mathrm{~s}^{-1}$ observed by the STEREO-A/PLASTIC instrument we have obtained a value of 1.1 AU for the length of the nominal Parker magnetic field line.

The vertical dashed lines in Fig. 2 indicate the onset times of type III radio bursts. It is clearly seen that there is a delay of 10 and $4 \mathrm{~min}$ between the detected in-situ spike and the first and second type III radio bursts, respectively. This delay can be partially explained if we take into account the intrinsic velocity dispersion in the energy range from 55 to $65 \mathrm{keV}$ and the background level, which can also mask the true spike onset. An enlarged Parker spiral in comparison with the calculated ideal length of 1.1 AU might also contribute to the observed delay (e.g. Kahler et al. 2011; Sáiz et al. 2005). Note that particles streaming along the Parker spiral were detected only with the sunward looking telescope indicating very low scattering in the interplanetary medium.

After 10:00 UT the electron intensity does not decrease to the pre-event level, but stays enhanced and represents a ramp with nearly constant intensity for more than one hour. Two reasons could be responsible for the formation of the ramp profile. First, there could be a continued injection of electrons from the source. This would be consistent with a long-lived jet observed by STA and SDO, which itself suggests the presence of open field lines, along which electrons can easily escape into interplanetary space for some time. Second, the spike beam could be additionally scattered by crossing of the IP shock shortly before the beam arrives at the spacecraft (not shown). This shock 


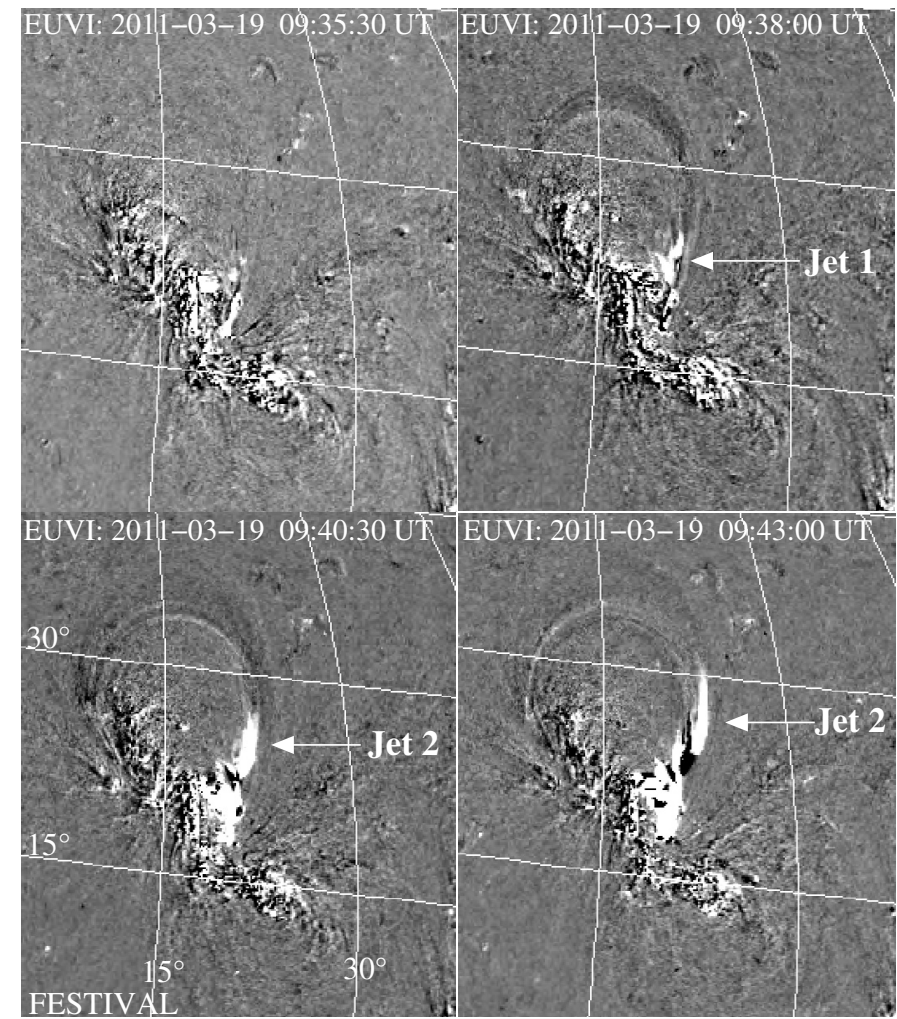

Fig. 3. Event on 19 March 2011: a sequence of EUVI/STA $195 \AA$ A running difference images showing two consecutive jets in the time interval 09:35:30-09:43:00 UT coinciding with the double type III burst and electron spike (see Fig. 2). All highly collimated jets expand northwards and each next jet is slightly shifted in the north-west direction in respect to the previous one. North is at the top, west is to the right.

overtook the spacecraft $1.5 \mathrm{~h}$ after the spike at 11:24 UT and was associated with a CME on 17 March.

Figure 3 presents a sequence of EUVI/STA $195 \AA$ running difference images showing at least two consecutive separate jets during the time interval from 09:35 to 09:43 UT coincident with the double type III burst and the electron spike (see Fig. 2). The footpoint of the jets slightly migrates towards north-west and appears progressively at higher altitudes. Therefore it is reasonable to assume that each subsequent jet migrates from closed field lines - loops inside the AR toward the border of the AR loop system and consequently towards the open field lines. In contrast to the slow migration speed the projected propagation speed of the jet towards the north as measured using the AIA/SDO $304 \AA$ images is sufficiently higher, $\sim 400 \mathrm{~km} \mathrm{~s}^{-1}$.

Since STEREO does not carry a magnetograph onboard it is not worthwhile to compare the magnetic configuration inferred from the EUV and radio observations with magnetic field extrapolations. But the existence of open field lines is demonstrated by the type III bursts.

Figure 4 shows the type III radio bursts source positions at different frequencies as observed by NRH in comparison to the EUV $304 \AA$ jet observations from the AIA/SDO. While the first AIA image (insert) was taken at 09:47 UT, a few minutes after the jet first appeared above the west limb, the second one was taken during the jet's largest extension at 10:29 UT (compare with EUVI/STA images, Fig. 3). We note, that for SDO the jet footpoint was located $\sim 25^{\circ}$ behind the west limb. At the $\mathrm{NRH}$ highest frequencies $(408-173 \mathrm{MHz})$ the source positions of both type IIIs at 09:36:06 and 09:40:47 UT match very well

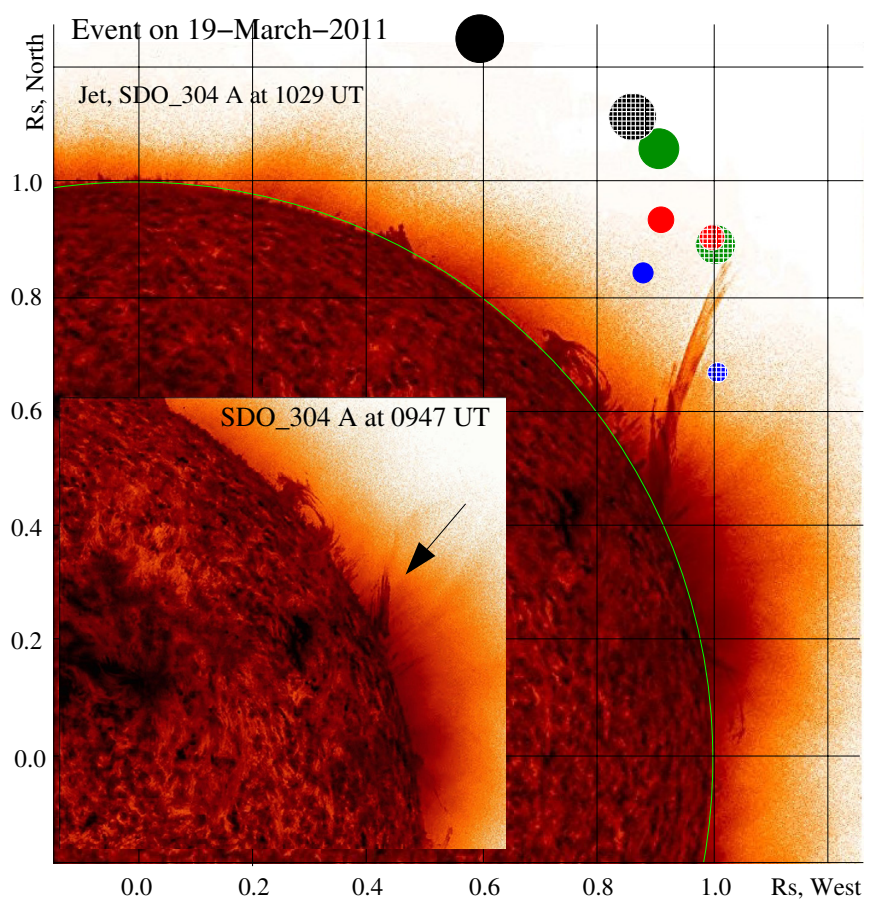

151 MHz: source at $093606 \mathrm{UT}$;

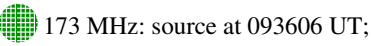

\# $270 \mathrm{MHz}$ : source at $093606 \mathrm{UT}$;

(1) $408 \mathrm{MHz}$ : source at $093606 \mathrm{UT}$;

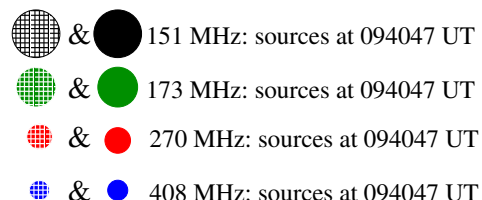

Fig. 4. Event on 19 March 2011. The same as in Fig. 3 jet observed by AIA/SDO $304 \AA$ in superposition of the metric type III radio sources obtained with Nançay Radioheliograph. Two images show the appearance and the evolution of the jet(s) between 09:47 and 10:29 UT. The jet footpoint was located $\sim 25^{\circ}$ behind the west limb from the SDO point of view NRH: Overlaid filled and hatched circles represent centroid positions of the double type III radio sources at frequencies between 408-151 MHz at 09:36:06 and 09:40:47 UT, respectively. The spatial coincidence of the type IIIs sources and the jet suggests the common origin of both phenomena. Solar north is at the top, west is to the right.

the location of the EUV jet. At the lowest frequency of $151 \mathrm{MHz}$ the source positions are considerably shifted towards the north. Obviously the type III electrons take a different path in comparison to the jet trajectory and escape towards the north along a specific magnetic flux tube connecting the spacecraft with the Sun. This spatial coincidence of the collimated jets and type III sources suggests that a hot coronal plasma and both, emitting radio emission and near-relativistic electrons of the spike were ejected, at least in the beginning, along the same diverging open magnetic field lines from the same source.

The observational results are summarized as following:

- A sequence of eight electron spikes streaming outwards from the Sun was detected on 26 February 2011 during a short time interval of $16 \mathrm{~h}$. All anisotropic spikes show velocity dispersion, almost symmetric time profiles, time durations below 12 min and occur simulateneously with type III radio bursts and EUV jets. Four of them show a delayed second peak of sunward streaming electrons suggesting a strong scattering (mirroring) beyond the STA orbit at a radial distance of 1.3 AU from the Sun, or $\sim 0.7 \mathrm{AU}$ along the magnetic field line beyond the s/c.

- Type III radio bursts accompanying the in-situ spike on 19 March 2011 reveal a close spatial and temporal 


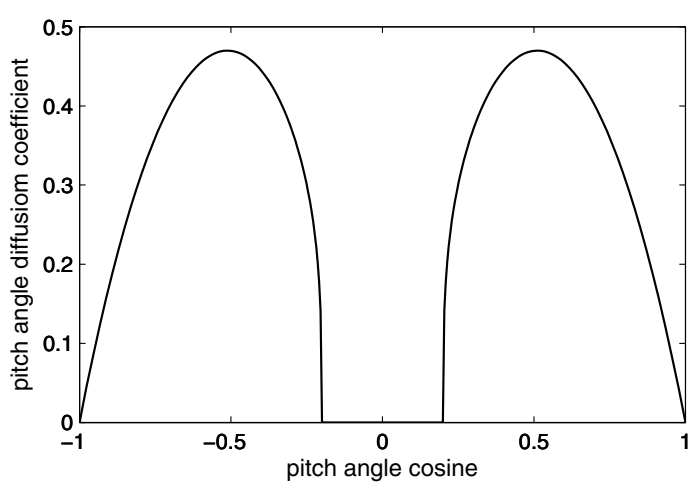

Fig. 5. Form of the pitch angle diffusion coefficient used in simulation of the 26 February 2011 spike.

coincidence with the very collimated EUV coronal jets suggesting a common origin.

- Close association between in-situ electron spikes, type III bursts and EUV jets implies a common origin of all these events due to the process of magnetic reconnection occurring in the solar atmosphere (e.g. Shibata 1992; Shimojo et al. 1996; Chifor et al. 2008) or due to electron acceleration by interaction of propagating coronal jets with surrounded plasma (Miteva et al. 2007).

- All presented events were detected during quiet solar wind conditions: slow solar wind speed $V_{\mathrm{sw}} \leq 405 \mathrm{~km} \mathrm{~s}^{-1}$, low density $\leq 2 \mathrm{~cm}^{-3}$, low proton temperature $\leq 1.5 \times 10^{5} \mathrm{~K}$ and smooth magnetic fields.

- The observed durations below $12 \mathrm{~min}$, the observed and the modeled spike-like time profiles (Sect. 3.3) reveal a limitation on particle injection/acceleration time into the interplanetary medium of a few minutes, which is comparable with the duration of the accompanying type III radio bursts.

\subsection{Spike modeling}

In the absence of large-scale disturbances such as CMEs and shocks, the interplanetary magnetic field can usually be described as a smooth average field, represented by an Archimedian spiral, with superimposed irregularities. In this case the propagation of charged particles consists of two components, adiabatic motion along the smooth field and pitch angle scattering off the irregularities. The quantitative treatment of the evolution of the particle's phase space density $f(s, \mu, t)$ can then be described by the model of focused transport (Roelof 1969):

$\frac{\partial f}{\partial t}+\mu v \frac{\partial f}{\partial s}+\frac{1-\mu^{2}}{2 L} v \frac{\partial f}{\partial \mu}-\frac{\partial}{\partial \mu}\left(D_{\mu \mu} \frac{\partial f}{\partial \mu}\right)=q(s, \mu, t)$.

Here $f(s, \mu, t)$ is the particle's phase space density, $s$ is the distance along the magnetic field line, $\mu=\cos \theta$ is the particle pitch angle cosine, $L(z)=B(z) /(-\partial B / \partial z)$ is the focusing length in the diverging magnetic field $B, D_{\mu \mu}(\mu)$ is the pitch angle diffusion coefficient and $t$ is the time. To model various types of pitch angle dependence for the diffusion coefficient we considered different types of the pith angle dependence of diffusion coefficient which had a gap around $\mu=0$, that is without scattering through 90 degrees.

The inclusion of such unusual form of pitch angle diffusion coefficient was done in accordance with (Dröge \& Kartavykh 2009). To model the electron spike time profiles on 26 February 2011 with onset at 16:03 UT we considered the
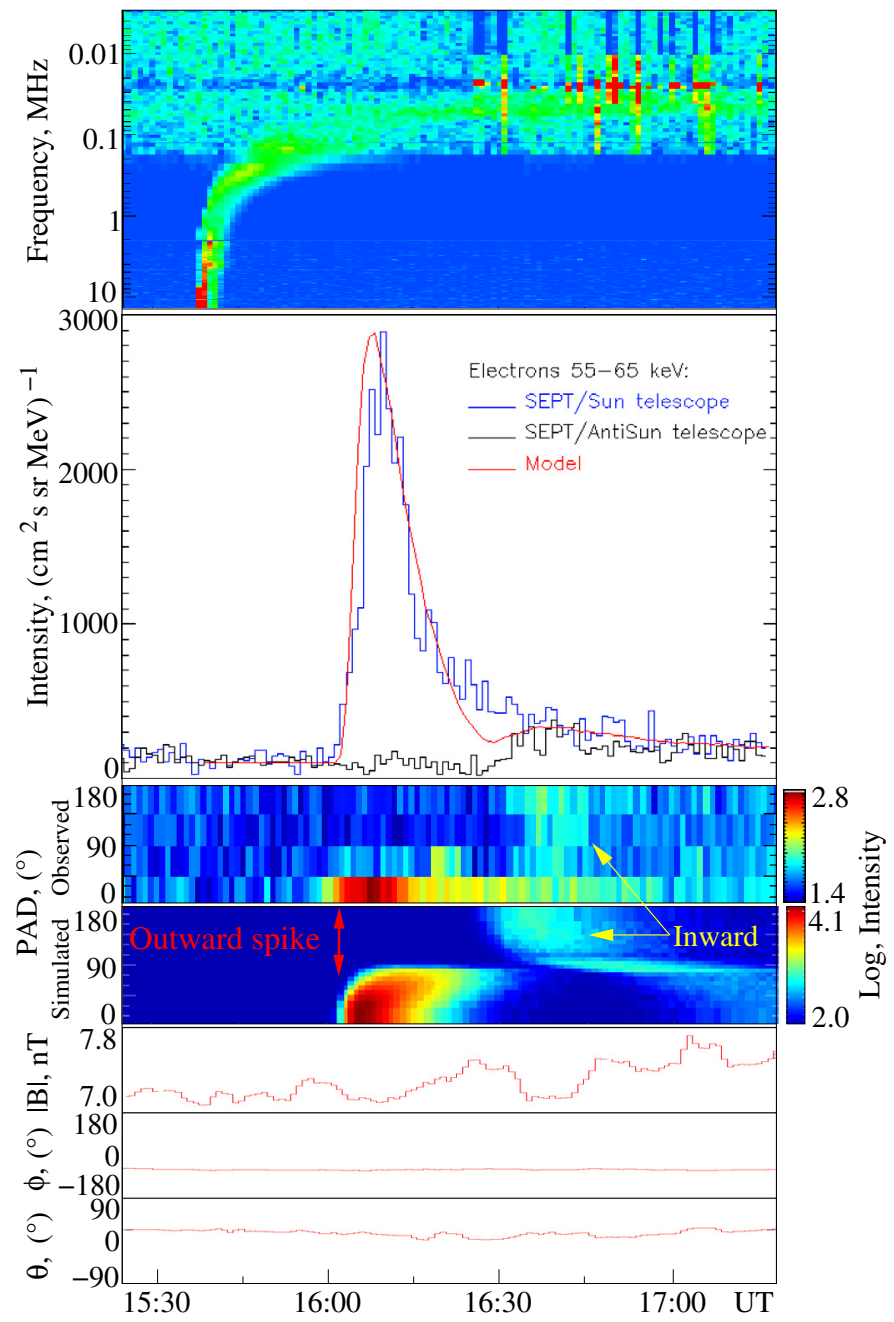

Fig. 6. Event on 26 February 2011. From top to bottom: (1) dynamic spectrum of the type III radio burst (SWAVES/STA), (2) a comparison between the observed spike intensity and the modeled omnidirectional time profile (red). Blue and black: time profiles of electrons streaming away and towards the Sun, respectively. (3) and (4) pitch angle distributions (PADs) of observed and simulated spike electrons, (5) magnetic field measured by MAG/STA. The observed time profiles and PAD are not shifted. A point-like source, an impulsive injection close to the Sun, and a reflecting boundary at a radial distance of $1.3 \mathrm{AU}$ from the Sun is considered. The diffusion coefficient is equal to zero if $|\mu| \leq 0.2$ (no scattering through 90 degrees, here $\mu$ is the pitch angle cosine).

solar wind speed of $300 \mathrm{~km} \mathrm{~s}^{-1}$ the heliocentric location of STEREO-A spacecraft of $0.96 \mathrm{AU}$ and used the pitch angle diffusion coefficient of the form shown in Fig. 5. This spike was chosen because it was observed with the best pitch angle coverage from all detected spikes. We note, that the SEPT instrument on non-spinning spacecraft has only four telescopes which often provide a very limited pitch angle coverage in comparison with spinning s/c. An impulsive injection and a point-like source at a radial distance of 0.05 AU magnetically connected with the spacecraft were assumed. The best coincidence of our simulations with the observations was obtained if the pitch angle diffusion coefficient had the form shown in Fig. 5 that is when the diffusion coefficient is equal to zero if $|\mu| \leq 0.2$. A reflecting boundary was considered at a radial distance of $1.3 \mathrm{AU}$ from the Sun and the probability of reflection was equal to 0.2 . This value is very close to the value of ratio of the peak intensities of inward and outward streaming electrons $(0.10-0.17)$. 
Figure 6 shows a comparison between the observed spike intensity (blue for outward and black for inward streaming/reflected electrons) and the modeled omnidirectional time profile (red). It is seen that our model is able to reproduce the shape of the main spike profile and its duration at FWHM, as well as the appearance of reflected electrons (black profile in Fig. 6) after 16:30 UT and the value of their maximal intensity.

The observed and simulated pitch angle distributions (PADs) are presented in panel 3 and 4, respectively. Both distributions are generally in agreement with each other and show a narrow anisotropic distribution between 0-45 degrees for the outward streaming and between 180-90 degrees for the inward streaming (reflected) electrons. Nonetheless there are some discrepancies between the simulation results and observations. Thus, at $\sim 16: 30$ UT the observed intensity is higher than the modeled one. This can be due to the assumption of the complete absence of scattering through 90 degrees. It would be more realistic to introduce a weak scattering in this region of $|\mu| \leq 0.2$, with a small finite value of $D_{\mu \mu}$. Another possibility to explain this discrepancy is to consider a narrow injection with tail. This would be in agreement with the prolonged ( $3 \mathrm{~min}$ ) type III radio emission.

There are also some discrepancies between the modeled and observed pitch angle distributions. First, after 16:45 UT there is an increase of intensity in the region $-0.2<\mu<0.2$ (pitch angle around 90 degrees). This effect comes directly from our model assumption about the absence of scattering for $|\mu|<0.2$. If the particle is thrown by a stochastic process in this region its pitch angle can be changed only by focusing, which is not strong far away from the Sun. Then the particle remains in the range of pitch angles with $-0.2<\mu<0.2$ for a long time. The fact that the observed PAD does not show such a feature is in favour of a finite scattering in the vicinity of 90 degrees pitch angle. Therefore the presented model serves as a first step, explaining the main properties of spikes.

Second, the modeled PAD is wider for electrons coming from the Sun, and narrower for electrons reflected back beyond the Earth. This can be due to two reasons. (a) SEPT has only four separate telescopes, and therefore, pitch angle distributions cannot be obtained with a high accuracy. (b) a suppression of scattering can be over larger interval of the pitch angle cosine.

\section{Discussion}

Our investigation confirms and extends the previous observations suggesting a very close temporal association between in-situ electron spike events, type III radio bursts and EUV jets. Additionally to previous studies we provide evidence that spikes are not only temporally correlated with type III radio emission and coronal jets but that there is a very close spatial coincidence between all these phenomena suggesting a common origin of these phenomena due to magnetic reconnection process occurring at the same location in the corona (e.g. Chifor et al. 2008) or due to electron acceleration in front of propagating coronal jets (Miteva et al. 2007). Moreover, since all spikes show similar profiles, the propagation conditions in the interplanetary medium should be not altered during time intervals up to $16 \mathrm{~h}$.

The jet and the type III radio sources on 19 March event show a very distinct and prolonged movement towards the north, i.e. in the direction opposite to the footpoint of the magnetic field line connected with the spacecraft STA. Assuming that nearrelativistic electrons take the same path as electrons responsible for the type III bursts it is not clear how these particles can arrive at the s/c? Evidently the magnetic field above the radio sources and the jet had a loop shape which presumably turned towards the ecliptic plane further away from the Sun and connects the injection source with the s/c. In this case the real electron propagation path could be longer than the Parker spiral length as calculated in Sect. 3.2, i.e. longer than 1.1 AU (see e.g. Kahler et al. 2011).

The modeled time profiles reproduce very well almost all features of the observed spike characteristics including the timing and the intensity of the reflecting electrons. An excess of the observed intensity over the simulated ones around 16:30 UT as well as an additional increase of particles density in PAD in the range $|\mu|<0.2$ can be explained by the absence of scattering through $\mu=0$.

All presented in-situ spikes show delay of 3-15 min from the associated type III bursts by assuming that the spikes electrons propagate along the nominal pathlength of about 1.2 $\mathrm{AU}$ from the Sun to the s/c. Consequently, assuming that the spike electrons and the type III radio burst emitting electrons were injected simultaneously, the real travel distance of 55-65 keV electrons should be around 1.4-1.8 AU, sufficiently larger than the nominal pathlength, and similar to the magnetic field line lengths of 1.6 AU as inferred from electron events by Kahler et al. (2011). This enlarged propagation distance inside of $1 \mathrm{AU}$ is not yet taken into account in the simulation, because the present model does not use any magneto-dynamical models of the solar wind which can essentially extend the pathlength (e.g. Ragot 2006).

\section{Summary}

The in-situ electron spikes detected at energies $\leq 300 \mathrm{keV}$ are characterized by very short durations (below 10-20 min at FWHM), almost symmetric time profiles, velocity dispersion and strong anisotropy revealing a weak particle scattering during the propagation from the Sun to 1 AU (STEREO). Using particle, EUV and radio imaging observations we prove, that electrons, forming the spikes, are accelerated during the same time and at the same location as the accompanying type III emitting electrons and narrow coronal EUV jets. Applying the particle propagation model we demonstrate that the characteristics of spikes reflect both, properties of the accelerator and the effects of interplanetary propagation.

Acknowledgements. The authors acknowledge the FESTIVAL and HELIOVIEWER teams that developed the browsers for manipulation of solar imaging data. We thank the STEREO/SWAVES/SECCHI/PLASTIC/MAG teams for their open data policy. Y.K. acknowledges support from the Russian Foundation for Basic Research (project No. 09-02-00019a). The STEREO/SEPT project is supported under grant 50 OC 0902 by the German Bundesministerium für Wirtschaft through the Deutsches Zentrum für Luft- und Raumfahrt (DLR). We thank our referee for helpful comments and suggestions.

\section{References}

Acuña, M. N., Curtis, D., Scheifele, J. L., et al. 2008, Space Sci. Rev., 136, 203 Agueda, N., Vainio, R., Lario, D., \& Sanahuja, B. 2010, A\&A, 519, A36 Aurass, H., Klein, K.-L., \& Martens, P. C. H. 1994, Sol. Phys., 155, 203

Bougeret, J. L., Goetz, K., Kaiser, M. L., et al. 2008, Space Sci. Rev., 136, 487 Chifor, C., Isobe, H., Mason, H. E., et al. 2008, A\&A, 491, 279

Dröge, W., \& Kartavykh, Y. Y. 2009, ApJ, 693, 6974

Dröge, W., Kartavykh, Y. Y., Klecker, B., \& Kovaltsov, G. A. 2010, ApJ, 709, 912

Ergun, R. E., Larson, D., Lin, R. P., et al. 1998, ApJ, 503, 435

Galvin, A. B., Kistler, L. M., Popecki, M. A., et al. 2008, Space Sci. Rev., 136, 437

Haggerty, D. K., \& Roelof, E. 2002, ApJ, 579, 841

Haggerty, D. K., \& Roelof, E. 2009, in Shock waves in space and astrophysical environments, ed. X. Ao, R. H. Burrow, \& G. P. Zank (AIP), 3

Howard, R. A., Moses, J. D., Vourlidas, A., et al. 2008, Space Sci. Rev., 136, 67 Innes, D. E., Cameron, R. H., \& Solanki, S. K. 2011, A\&A, 531, L13 
A. Klassen et al.: Solar origin of in-situ electron spikes

Kahler, S., Aurass, H., Mann, G., \& Klassen, A. 2007, ApJ, 656, 567

Kahler, S. W., Haggerty, D. K., \& Richardson, I. G. 2011, ApJ, 736, 106

Kerdraon, A., \& Delouis, J. 1997, The Nançay Radioheliograph, in Lect. Notes

Phys., Coronal Physics from Radio and Space Observations, ed. G. Trottet (Springer), 483, 192

Klassen, A., Bothmer, V., Mann, G., et al. 2002, A\&A, 385, 1078

Klassen, A., Gómez-Herrero, R., \& Heber, B. 2011, Sol. Phys., 273, 413

Klein, k.-L., Krucker, S., Trottet, G., \& Hoang, S. 2005, A\&A, 431, 1047

Krucker, S., Larson, D. E., Lin, R. P., \& Thompson, B. J. 1999, ApJ, 519, 864

Krucker, S., Kontar, E. P., Christe, S., Glesener, L., \& Lin, R P. 2011, ApJ, 742, 82

Lemen, J. R., Title, A. M., Akin, D. J., et al. 2012, Sol. Phys., 275, 17

Lin, R. P. 1974, Space Sci. Rev., 16, 189

Lin, R. P. 1985, Sol. Phys., 100, 537

Miteva, R., Mann, G., Vocks, C., \& Aurass, H. 2007, A\&A, 461, 1127
Müller-Mellin, R., Böttcher, S., Falenski, J., et al. 2008, Space Sci. Rev., 136, 363 Nitta, N., Reames, V., DeRosa, M. L., et al. 2006, ApJ, 650, 438

Ragot, B. R. 2006, ApJ, 653, 1493

Raulin, J. P., Kundu, M. R., Hudson, H. S., Nitta, N., \& Raoult, A. 1996, A\&A, 306, 299

Roelof, E. C. 1969, in Lectures in High-Energy Astrophysics, ed. H. Ögelman, \& J. R. Wayland (Washington, DC: NASA), NASA SP-199, 111

Sáiz, A., Evenson, P., Ruffolo, D., \& Bieber, J. W. 2005, ApJ, 626, 1131

Shibata, K., Ishido, Y., Acton, L. A., et al. 1992, PASJ, 44, L173

Shimojo, M., Hashimoto, S., Shibata, K., et al. 1996, PASJ, 48, 123

Suzuki, S., \& Dulk, G. A. 1985, in Solar Radiophysics, ed. D. J. McLean, \& N. R. Labrum (Cambridge University Press), 289

Wang, Y.-M., Pick, M., \& Mason, G. M. 2006, ApJ, 639, 495

Wang, L., Lin, R. P., \& Krucker, S. 2011, ApJ, 727, 121 